\title{
Memristive-biosensors: A new detection method by using nanofabricated memristors
}

\author{
Sandro Carrara ${ }^{\mathrm{a}, *}$, Davide Sacchetto ${ }^{\mathrm{a}}$, Marie-Agnès Doucey ${ }^{\mathrm{b}}$, Camilla Baj-Rossi $^{\mathrm{a}}$, \\ Giovanni De Micheli ${ }^{a}$, Yusuf Leblebici ${ }^{a}$ \\ a EPFL - École Polytechnique Fédérale de Lausanne, Switzerland \\ ${ }^{\mathrm{b}}$ UNIL - Université de Lausanne, Switzerland
}

\section{A R T I C L E I N F O}

\section{Article history:}

Received 15 November 2011

Received in revised form 4 April 2012

Accepted 13 April 2012

Available online 11 May 2012

\section{Keywords:}

Biosensor

Silicon nanowire

Memristive effect

Schottky barrier

Polyclonal antibodies

\begin{abstract}
A B S T R A C T
This paper proposes a new detection methodology based on memristive-effect registered on silicon nanowire. The nano-wires are fabricated by a lithographic technique that allows precise and selective etching at the nanoscale. The wires are obtained in three main steps. Initially, a photoresist line defines the wire position. In a second step, silicon deep reactive ion etching is performed to obtain a scalloped trench. In the final step, the trench is reduced to a suspended nanowire after wet oxidation. The obtained wires present Schottky barrier contacts and are used for bio-molecular detection on dried samples. The memristive silicon nanowire devices are functionalized with rabbit antibodies in order to sense antigens. The sensitivity and detection limit of this new kind of nano-bio-sensors are estimated equal to $37 \pm 1 \mathrm{mV} / \mathrm{fM}$ and $3.4 \pm 1.8 \mathrm{fM}$, respectively.
\end{abstract}

(C) 2012 Elsevier B.V. All rights reserved.

\section{Introduction}

Memory effects are largely used in nature for several aims. The brain gather, store and retrieve any kind of information in synaptic connections [1]. Side-chains polymers store mechanical information in shape [2] and, thus, adaptive chemistry suggests memory effects in molecular organization [3]. As well known, computers store electrical information in capacitors, transistors, and hard disks. We usually need of complex circuits involving transistors for storing information in electrical charges. Transistors are three-terminal elements while Leon Chua defined in 1971 a reliable two-terminal single-device being capable of memory effect by trapping charges in flux-linkage [4]. Leon Chua called this single-device with the name of memristor. However, he used complex circuits based on BJT (Bipolar Junction Transistors), FET (Field Effect Transistors), and Operational Amplifiers to obtain an electrical device actually working as a memristor [4]. Only in 2008 Stanley Williams reopened the question by showing that many structures fabricated at the nanoscale exhibit on ions migration and behave as the

\footnotetext{
* Corresponding author.

E-mail address: sandro.carrara@epfl.ch (S. Carrara).
}

memristor identified by Leon Chua in 1971 [5]. Several different nanofabricated devices, such as memcapacitors and meminductors [6], manifest memory effects similar to that described by Chua and Williams. In all these structures, the memory effect depends on charge carriers rearrangement at the nanoscale as due to external perturbations [6]. These memory-effect devices have been fabricated by using different materials, including amorphous silicon [7], crystalline silicon [8], platinum/ $/ \mathrm{TiO}_{2}$ [9], platinum/organic-films [5], aniline-derivatized conductive-polymers [10], and graphene embedded in insulating polymers [11]. These devices have been proposed for different applications including digital [9] and ana$\log$ [12] memories, logic [13] and neuromorphic [14-16] circuits. Although nanowires have been proposed several times as gas sensors [17], and as ion-sensitive Field Effect Transistor (FET) for cancer markers [18] or DNA [19] detection, memristive effect has never been reported before as actually applied for biosensing. The aim of this paper is to report a new bio-detection based on memristive signals acquired on silicon freestanding nano-wires. We define as memristive-biosensors these nano-devices. We verify with Scanning Electron Microscopy the fabricated and bio-functionalized nanostructures. We report acquisitions of conductivity on such structures that clearly show memristive behavior. We show that both probe antibodies and target antigens affect the memristive behavior. We show that the humidity affects our memristive 
signals. Finally, we calibrate the biosensors under controlled humidity and temperature. We successfully demonstrate the feasibility of this conceptually new bio-detection in the fempto-molar range of concentrations.

\section{Materials and methods}

\subsection{Memristors nano-fabrication}

Silicon on insulator substrates with low boron concentration $\left(N_{\mathrm{A}} \approx 10^{15}\right.$ atoms $/ \mathrm{cm}^{3}$ ) have been used (Fig. $1 \mathrm{~A}$ ) to fabricate the memristive-biosensors. A hard mask layer composed by $20 \mathrm{~nm}$ of dry oxide and by $80 \mathrm{~nm}$ of "low stress" $\mathrm{Si}_{3} \mathrm{~N}_{4}$ silicon nitride is formed by thermal oxidation and low-pressure chemical vapor deposition (LPCVD), respectively (Fig. 1B). Then, optical lithography with $1 \mu \mathrm{m}$ resolution defines $10 \mu \mathrm{m}$ and $20 \mu \mathrm{m}$ long photoresist lines that are used for mask layer patterning with inductively coupled plasma (ICP) (Fig. 1C). Suspended Si nanowires are thus defined by isotropic ICP Si etching with fluorine chemistry, thanks to an undercut formation below the $\mathrm{SiO}_{2} / \mathrm{SixNy}$ hard mask (Fig. 1D). By optimizing the etching undercut, suspended sub-lithographic Si ribbons attached to Si pillars are formed with cross-sectional dimensions of $600 \mathrm{~nm}$ diameter, as confirmed by SEM imaging (see Section 3). A sacrificial thermal oxidation process is then carried out with the double purpose to eliminate the $\mathrm{Si}$ in excess and to reduce the surface roughness induced by the etching. Starting from wet oxide growths, most of the Si ribbon is consumed and suspended Si nanowires are formed (Fig. 1E). Then, contact sites are defined as apertures into the hard mask layer and, subsequently, native $\mathrm{SiO}_{2}$ is removed by dipping the substrate into a buffered hydrofluoridric acid solution to obtain a good quality surface. Just after native oxide removal, the substrate is loaded in vacuumed evaporator to avoid a thick native oxide re-growth. Next, Ni/Ti bylayer of $50 \mathrm{~nm} / 10 \mathrm{~nm}$ thicknesses are evaporated on the substrates to form a metallic junction (Fig. 1G) in the location where the hard mask has been opened. Finally, the sample is annealed at low temperature in nitrogen $\mathrm{N}_{2}$ atmosphere to obtain stoichiometric $\mathrm{NiSi}$ $1: 1$ silicide phase junctions and pads for electrical characterization. Un-reacted metal is removed by Piranha solution (a proper mixture of sulfuric acid $-\mathrm{H}_{2} \mathrm{SO}_{4}$ and hydrogen peroxide $-\mathrm{H}_{2} \mathrm{O}_{2}$ ) heated at $100{ }^{\circ} \mathrm{C}$ for $10 \mathrm{~min}$ (Fig. $1 \mathrm{H}$ ). The cleaning with piranha solution also serves as surface treatment for bio-molecule grafting [20]. The so-fabricated memristive-biosensors are, then, functionalized with protein probes as described in Section 2.2 (Fig. 2).

\subsection{Memristors bio-functionalization}

The surface of the substrates is initially soaked again in piranha solution. Piranha solution results in a highly hydrophilic silicon surface and sets $\mathrm{OH}$ groups into the surface. $\mathrm{OH}$ groups are suitable for covalently binding of the proteins [21]. The silicon channels are then functionalized by covalent attachment of rabbit polyclonal antibodies with GPTS (glycidoxypropyltrimethoxysilane) [22] by using a modification of the procedure described by Kim et al. [23]. The silicon nanowire surface is incubated for $60 \mathrm{~min}$ at RT (Room Temperature) in ethanol containing $10 \mathrm{mM}$ acetic acid and 1\% GPTS. Following extensive washes with ethanol/acetic acid solution, the surface is dried under a $\mathrm{N}_{2}$ stream and placed for $15 \mathrm{~min}$ at $110^{\circ} \mathrm{C}$ in a dried oven. The surface is then cooled down at RT and incubated over night in a humid chamber at RT with PBS (Phosphate Buffer Saline) solution containing $0.5 \mathrm{mg} / \mathrm{ml}$ of polyclonal rabbit antibodies. Following extensive washes with PBS, the remaining active GPTS-derived groups are blocked by ethanolamine ( $10 \mathrm{mM}$ ethanolamine at $\mathrm{PH} 8.0$ ) for $60 \mathrm{~min}$ at RT. The excess of ethanolamine is removed by PBS washes and the surface blocked by an additional incubation with PBS containing $3 \%$ gelatin from cold water fish skin for $30 \mathrm{~min}$ at RT. The modified surface is washed again and stored in PBS at $4{ }^{\circ} \mathrm{C}$ until use. All the used chemicals were supplied by Sigma-Aldrich (St-Louis, MO).

\subsection{SEM imaging}

Morphological analysis of the memristive-biosensors is performed by using a SEM Microscope from Zeiss, model Leo 1550. In order to verify the nanofabrication process, SEM images are acquired on $\mathrm{Si}$ ribbons having $600 \mathrm{~nm}$ trench dimension before sacrificial oxidation, on the suspended nanowires having $400 \mathrm{~nm}$ diameter and NiSi junctions, and on the nanowires with NiSi silicided extremities after the metal is stripped using a $100{ }^{\circ} \mathrm{C}$ hot Piranha etching step. A final imaging is done after bio-functionalization. A $4 \mathrm{~nm}$ layer of $\mathrm{OsO}_{4}$ is sputtered onto biofunctionalized nanowires in order to avoid the protein charging effect and, therefore, to provide contrast to images. The accelerating voltage is equal to $4 \mathrm{kV}$. In some cases, the stage holder is tilted at $14.3^{\circ} \mathrm{C}$ or at $27.5^{\circ} \mathrm{C}$ in order to acquire more clear pictures of the nanostructures.

\subsection{Bio-detection measurements}

The functionalized memristive-biosensors are exposed to antigens (anti-rabbit antibodies) for $2 \mathrm{~h}$ at RT in PBS at the indicated concentrations. The excess of antigen is removed by washing the substrates surface three times with PBS. The surface is dried by using an air gun and immediately used for measuring the antibody-antigen interaction. The samples are not measure in liquid conditions but in dried ones. Several experiments are conducted by varying humidity and temperature for a deeper understanding of the effects of physical parameters on the memristive behavior. The memristive curves are acquired by using the following equipments: Hewlett-Packard 4156A precision semiconductor parameter analyzer; Agilent Technologies Integrated Circuit Characterization and Analysis Program; Cascade Microtech Probe station; Nucleus Probe Station Control Software; Rotronic HC2-C04 Thermo-Hygrometer. The substrates are placed on top of a conductive-chuck enabling the application of a potential to the devices' backside. The backside potential was usually kept at $0 \mathrm{~V}$ with respect to the source electrode. This is done to avoid floating backside gate effects. Current/voltage curves are acquired at different voltage scan rate to verify memristive effect and, then, the swap rate is $10 \mathrm{mV} / \mathrm{s}$ is selected for memristive detection. Every time, each device is measured by following the same sequence: non-functionalized sample, functionalized sample, functionalized sample after antigen uptake at different concentrations.

\section{Results and discussion}

\subsection{Memristors at the nanoscale}

As previously anticipated, the nanofabrication and the antibody grafting are followed with SEM microscopy to check any crucial step. As showed in Fig. 3, suspended sub-lithographic Si ribbons attached to Si pillars are formed with cross-sectional dimensions of $600 \mathrm{~nm}$ diameter after optimizing the undercut etching. Fig. 4 shows the quality of the apertures required into the hard-mask passivation-layer in order to fabricate the memristive-biosensor contact sites. Fig. 5 shows the sample after the annealing at $400^{\circ} \mathrm{C}$ in nitrogen $\mathrm{N}_{2}$ atmosphere to obtain the silicide phase required to form junctions and pads for the electrical characterization. The image reported in Fig. 5 was obtained after the treatment with Piranha solution. Fig. 5 reports the structure immediately before surface treatment with the proteins, while Fig. 6 shows 


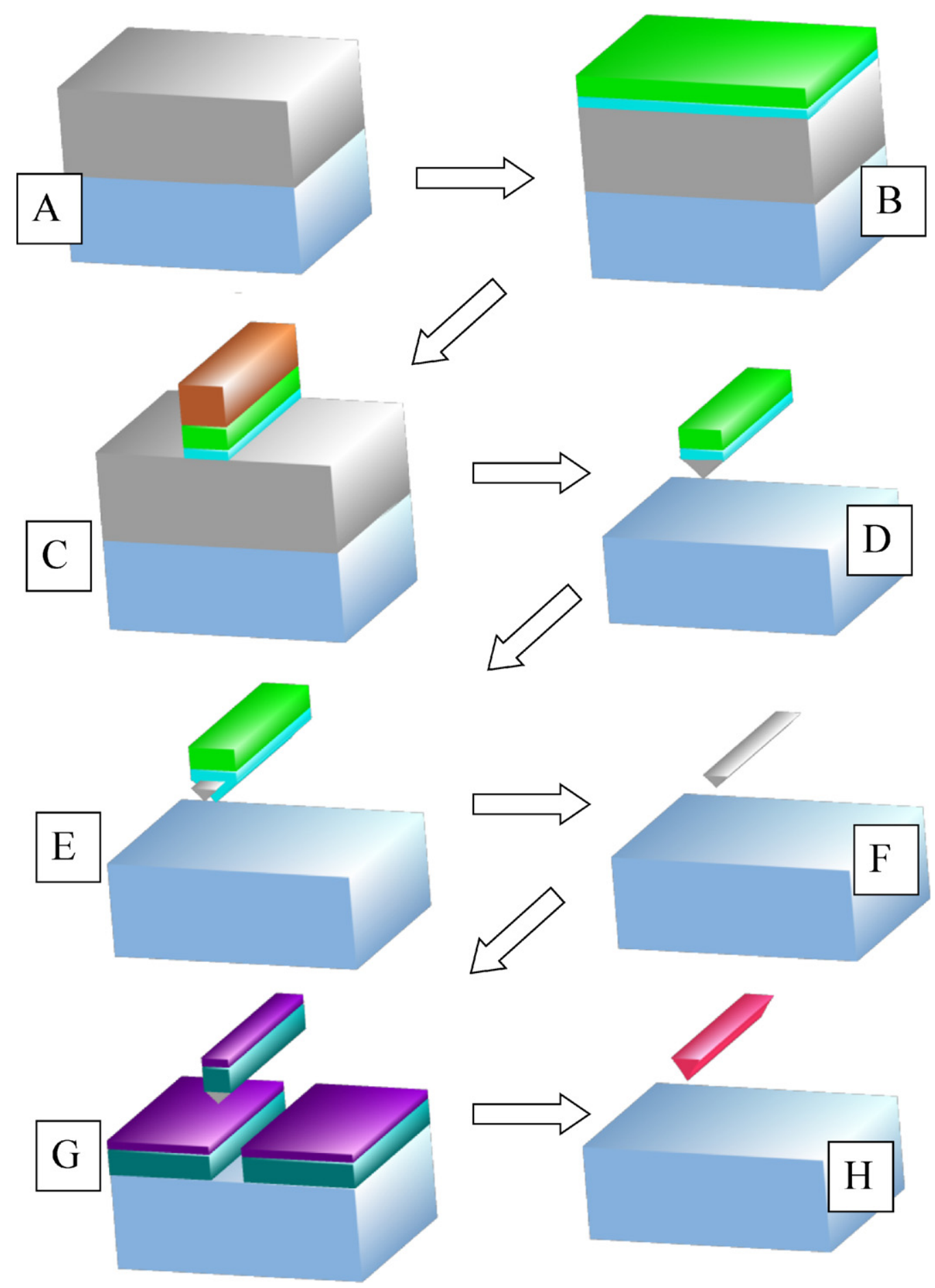

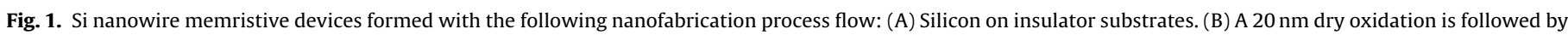

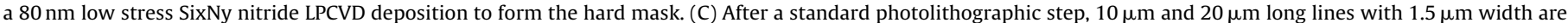

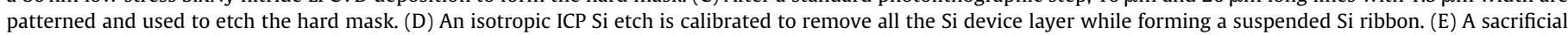

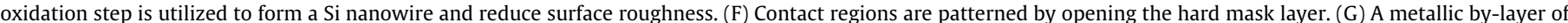

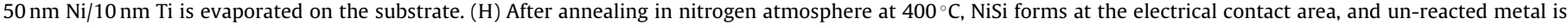
removed by Piranha etching. The devices are now ready for the bio-functionalization.

the memristive-biosensors just after the functionalization with the antibodies. SEM images show the memristive-biosensor at this stage of the preparation with feature very similar to those in Fig. 5. The SEM imaging does not distinguish organic material with respect to silicon. However, we can analyze the SEM images before and after the bio-functionalization and compare the increased diameters with sizes expected by the bio layer. The nanowires before the functionalization with antibodies (Fig. 5) presents a size equal to $483 \pm 51 \mathrm{~nm}$, while the same measure done on the functionalized wires (Fig. 6) returns a value of $582 \pm 36 \mathrm{~nm}$. The expected theoretical size of a single layer of antibodies [24] is estimated in $10.4 \pm 9.3 \mathrm{~nm}$ while the difference in the wires diameter in SEM images returns a larger value. However, several considerations may be done about this comparison. The anchoring of antibodies onto 


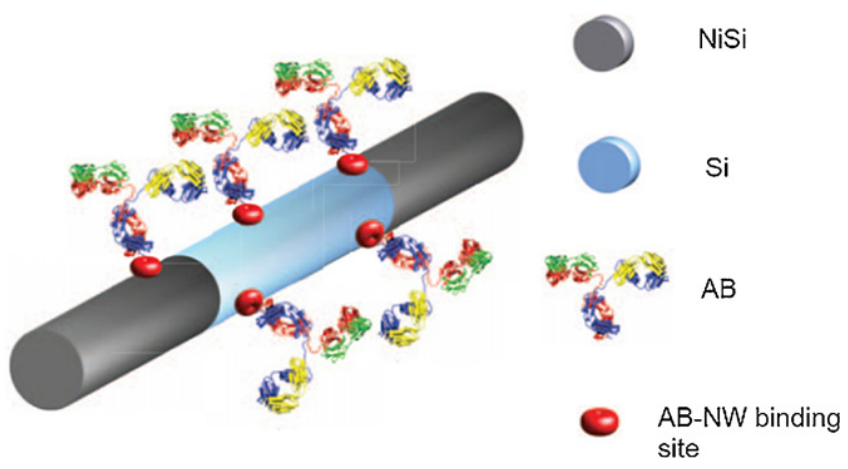

Fig. 2. Si nanowire with NiSi contacts after silicidation and after functionalization with anti-rabbit polyclonal antibodies (AB).

GPTS is provided by $\mathrm{SH}, \mathrm{NH}_{2}$, and $\mathrm{COOH}$ groups on the proteins. The anchoring with the $\mathrm{NH}_{2}$ groups is the most efficient binding. $\mathrm{NH}_{2}$ groups are contained in Lysines and Arginines, which are widely spread over the entire antibody' surface [25]. So, randomly orientated antibodies are considered to estimate the size of antibodies layer. The random orientation of proteins also explains the large error estimated for the layer size. However, both antibodies and GPTS provide the bio-functionalization layer (see Section 2.2) and, moreover, gelatin is added to block un-reacted anchoring groups. Finally, we cannot be sure to get monolayers of antibodies due to protein aggregation at the $\mathrm{pH}$ used for the functionalization. So, the final bio-layer size is expected larger than that of a single antibodies layer. The value of $582 \mathrm{~nm}$ registered on the functionalized wires needs to be reduced by $8 \mathrm{~nm}$ due to the layer of $\mathrm{OsO}_{4}$ required to get enough contrast on the biomaterials (see Section 2.3). Most important, the large errors found in estimations from SEM images are due to the nano-fabrication process on the wires. Therefore, we can conclude that the increasing of the apparent wires diameter is coherent with the presence of the bio layer.

\subsection{The innovative memristive bio-detection}

As usual in measuring nanowires, we fix the potential on backside of the substrate in order to avoid floating potentials at the nanostructure. In that manner, the substrate acts as back gate as well as in the usual FET configurations of nanowires. We acquire the current flow between the two terminals of our memristivebiosensors as the source-drain current $I_{\mathrm{ds}}$ versus the potential

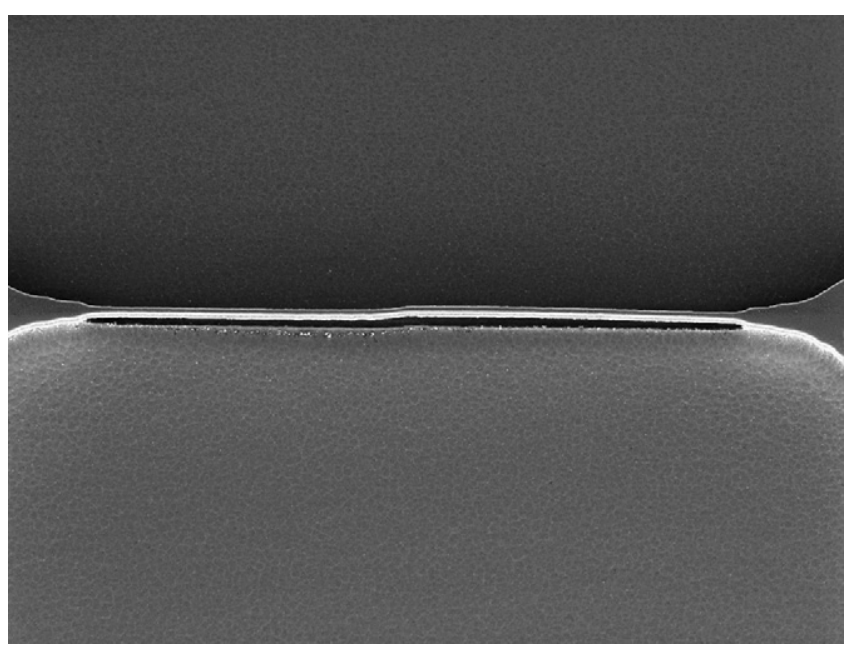

Fig. 3. Suspended Si ribbon with $600 \mathrm{~nm}$ trench dimension before sacrificial oxidation.

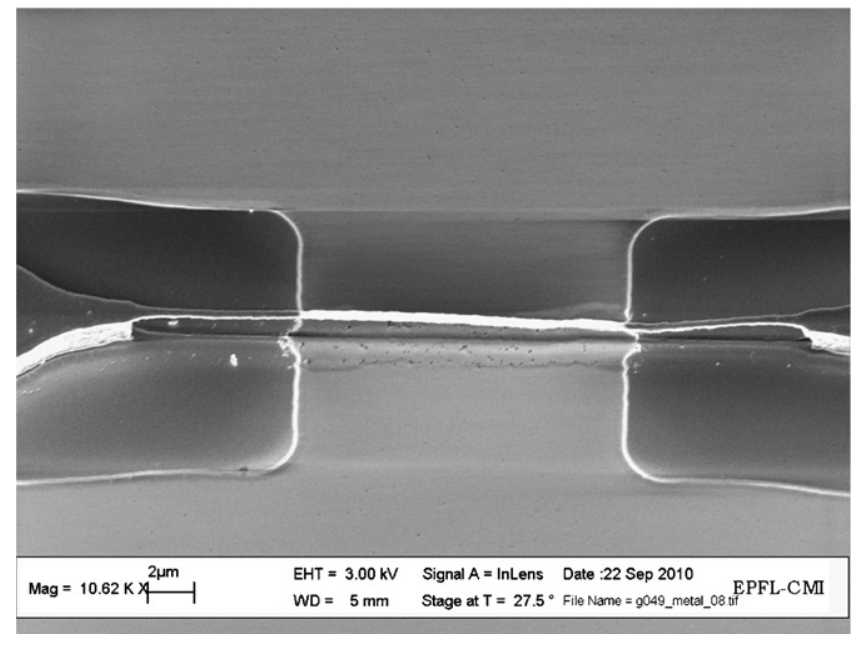

Fig. 4. Suspended Si nanowire and hard mask opened at contact regions before Ni/Ti metal deposition.

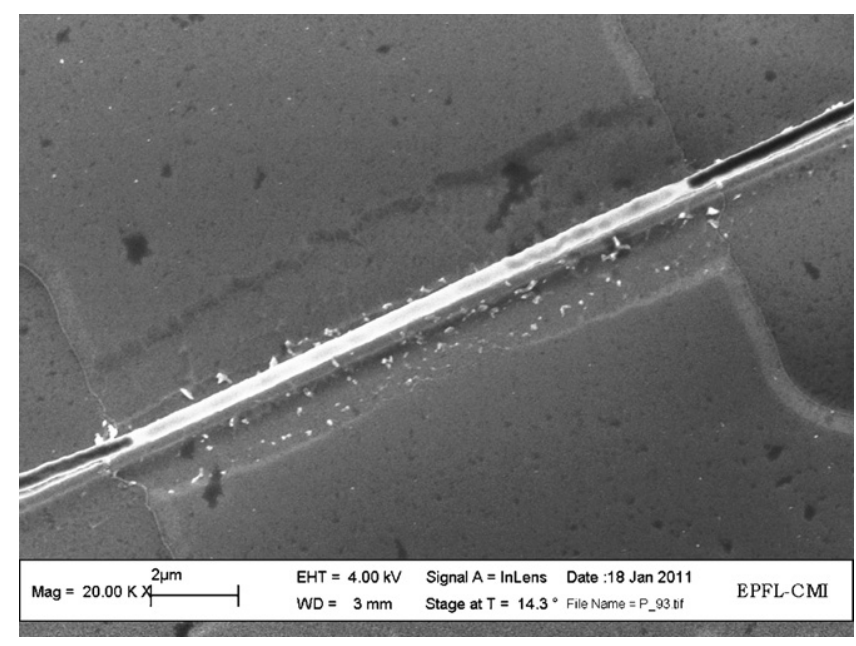

Fig. 5. Si memristive-biosensors with NiSi silicided extremities after the un-reacted metal is stripped using a $100^{\circ} \mathrm{C}$ hot Piranha etching step. After this step the devices are ready to be functionalized with bio-molecules.

applied to these terminals. We refer to this bias potential as the $V_{\mathrm{ds}}$ in order to use the very-well established terminology usually used for nanowire-based FET transistors. Under these assumptions, the acquisition of the memristive behavior is seen in terms of $I_{\mathrm{ds}}$

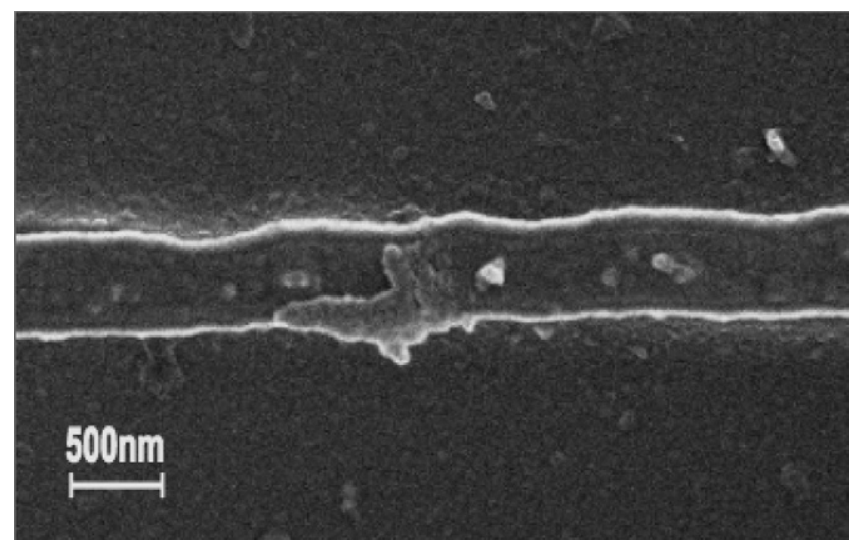

Fig. 6. Si memristive-biosensors functionalized by covalent attachment of rabbit polyclonal antibodies. After this step the devices are ready to be used for detecting the antigens. 


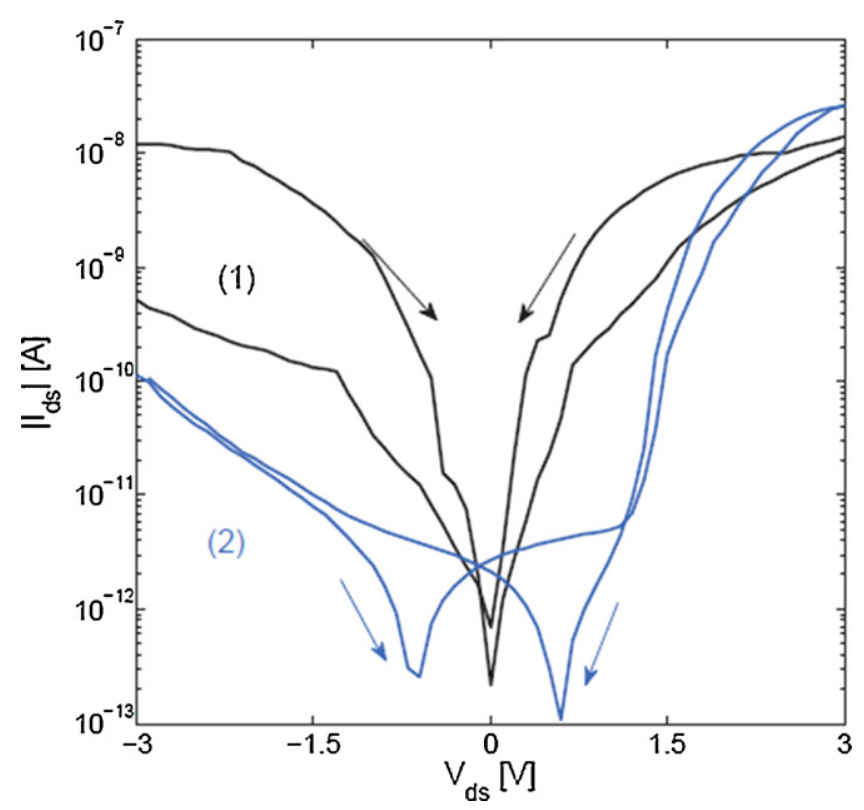

Fig. 7. $I_{\mathrm{ds}}-V_{\mathrm{ds}}$ curves taken (1) before and (2) after memristive-biosensors functionalization with an all-around $\mathrm{AB}$ layer demonstrating the origin of a current minima gap with the presence of a bio-molecular layer.

versus the bias potential $V_{\mathrm{ds}}$. Fig. 7 clearly shows that the functionalization with probe antibodies definitely changes the memristive behavior of the structure. One of the distinctive features of any memristive system is a pinched frequency-dependent hysteresis loop [26]. In fact, we have observed that the acquired hysteresis is depending on the bias sweep rate of the applied signals and, in particular, it was wider for diminishing scan rates while it was close for very fast scan rates. The curve (1) corresponds to the conductivity of the memristive-biosensor without any protein onto the silicon freestanding channel. In the forward and backward branches of $I_{\mathrm{ds}} / V_{\mathrm{ds}}$ curve, the graph shows a memristive effect as encoded by different current values for the same bias value. For example, the current is lower of more than one order of magnitude at $V_{\mathrm{ds}}$ equal to $-3 \mathrm{~V}$ in the backward than in the forward scan. However, the most innovative change after bio-functionalization is the wide difference in the bias used for reaching current minima.
Curve (2) in Fig. 7 shows that the current reaches the minimum at $-0.6 \mathrm{~V}$ during the forward branch and at $+0.6 \mathrm{~V}$ in the backward one. This means a voltage gap is created by the protein functionalization as a further memory effect on the voltage scan across the nanowire. No voltage gap is present in curve (1). To understand this phenomenon, we can consider the electrical conditions of the biosensor. Any protein is composed of hydrophobic and hydrophilic charged residues. Under correct physiological conditions ( $\mathrm{pH} 7.4$ ), arginine and lysine residues are positively charged while aspartic and glutamic acids are negatively charged. In any antibody (Fig. 8A), positively charged residues (Fig. 8B) are in excess with respect to negatively charged ones (Fig. $8 \mathrm{C}$ ), even if the charge distribution is quite similar. So, the presence of antibodies all-around the freestanding nanowire contributes to extra charges surrounding the channel. The net contribution of their positively charged residues acts by creating an electrical field surrounding the channel of our memristive-biosensor. The effect we get from this virtual all-around bio-gate is equivalent to that we have in case of nanostructures without any bio-functionalization but fabricated with an all-around silicon gate [27] (Fig. 9). In fact, the all-around gates give to those structures the memristive conductivity showed in Fig. 9(A). The bias voltage applied to the all-around gate (defined here as $V_{\mathrm{gs}}$ ) provides now the voltage gap. This gap is larger for $+3 \mathrm{~V}$ than for $-3 \mathrm{~V}$ in $V_{\mathrm{gs}}$. Back to Fig. 8, we see that the antibodies provide more positive charges than negative ones. This proofs that the virtual all-around gate provided by proteins provides the voltage gap.

The antigens detection also affects the voltage gap created by the antibodies. This gap varies after the antigen uptake, as clearly shown in Fig. 10. Comparing curves (1) and (2) of the figure, we see that the current does not always transduce the memristic effect due to antigen up-take. At $-1 \mathrm{~V}$, the curve (1) and the curve (2) almost show the same current difference between the forward and the backward branches. In the forward branches of curves (1) and (2), the two currents are almost identical at $-0.5 \mathrm{~V}$. Before antigen uptake, the forward current at $+1 \mathrm{~V}$ is larger than that the backward one, while it is the opposite after the uptake of antigens. The reason of such non-reproducible memristive effect on the current is also related to parasitic resistances on contacts of the device pads. Indeed, the voltage gap encodes very well the antigens interactions. In fact, the current gap coherently decrease with increasing amount of antigens, as demonstrated by curves (2) and (3). Fig. 10 clearly shows highly different currents in the three showed curves while it shows a clear trend dealing with the voltage gap reached by the

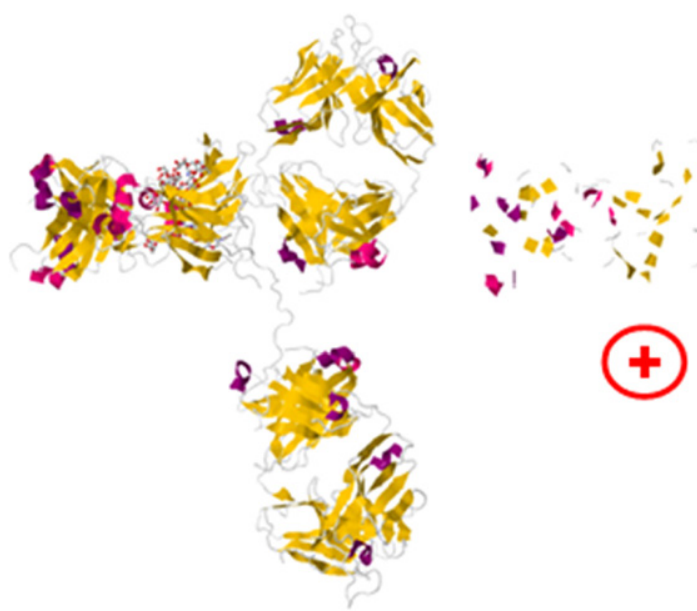

(A)

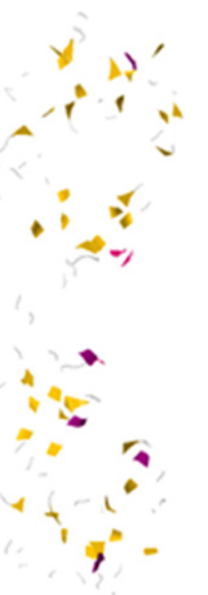

(B)

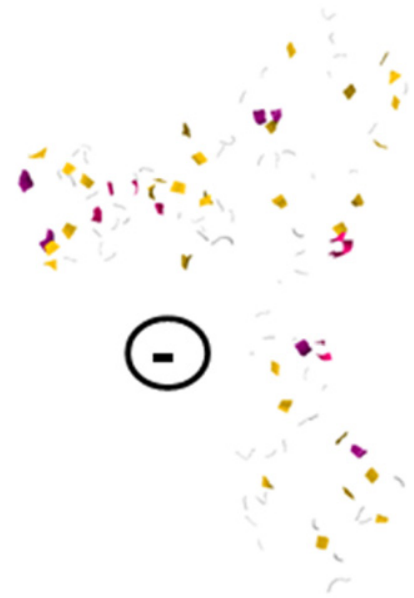

(C)

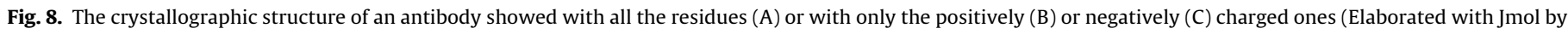
BDP structures data bank, on the antibody structure related to Ref. [22]). 


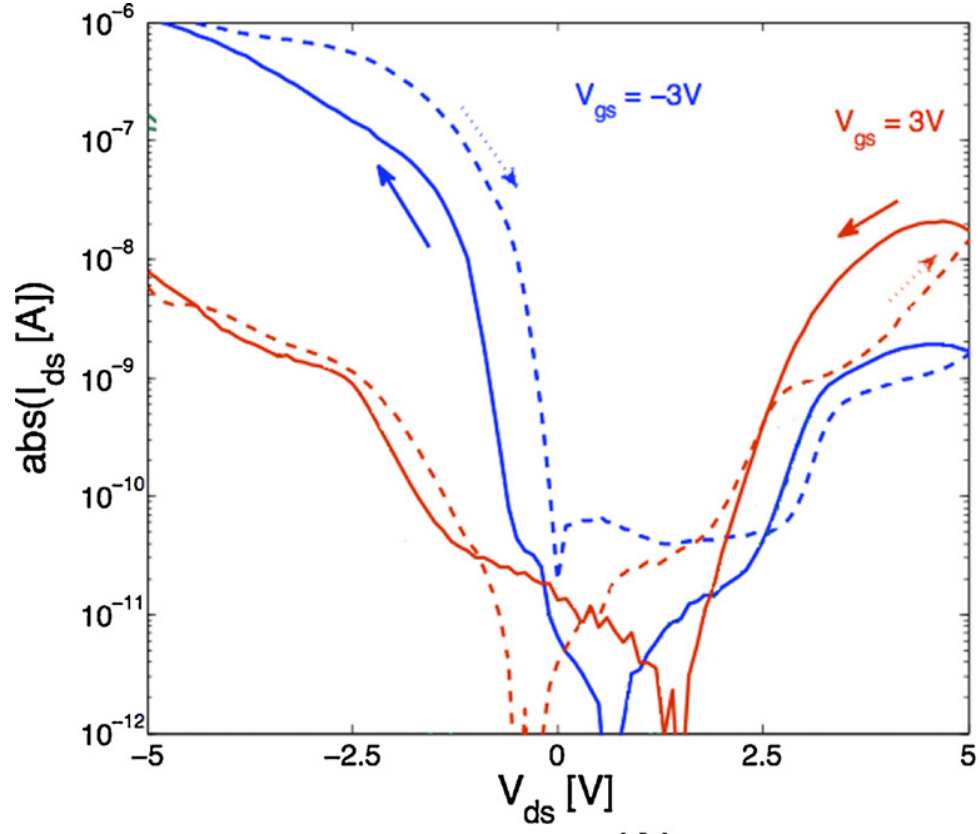

(A)

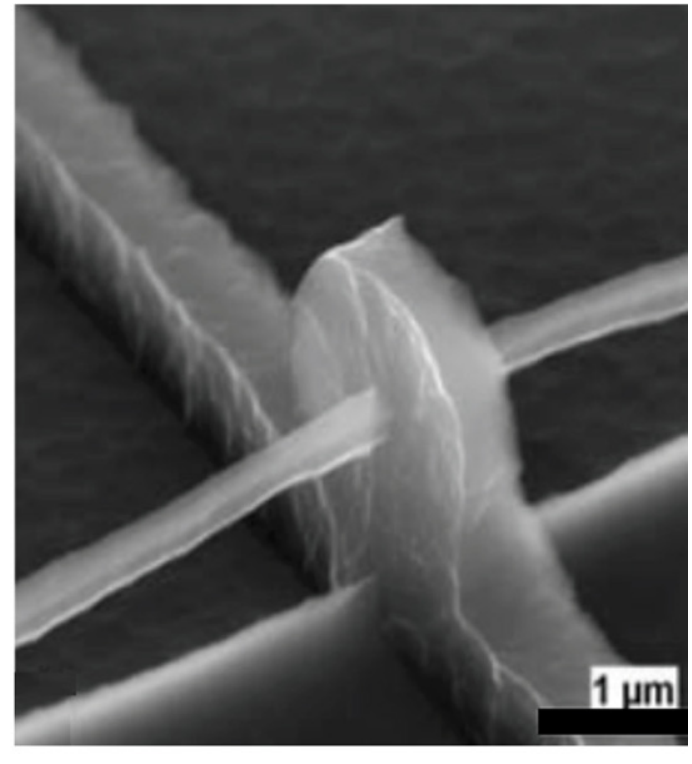

(B)

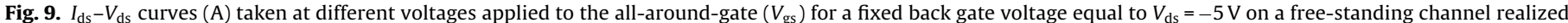
onto a silicon-on-insulator substrate with an all-around gate (B).

current minima. So, the memristive behavior of the nanostructure is registered in a coherent and reproducible manner by the voltage gap.

\subsection{Calibration of the memristive-biosensors}

If we now define the voltage gap as the measure of the antigens uptake, then we can obtain a new detection method. It is worth noting that this voltage gap is diminishing with increasing antigens concentrations. The phenomenon of a voltage gap that diminishes

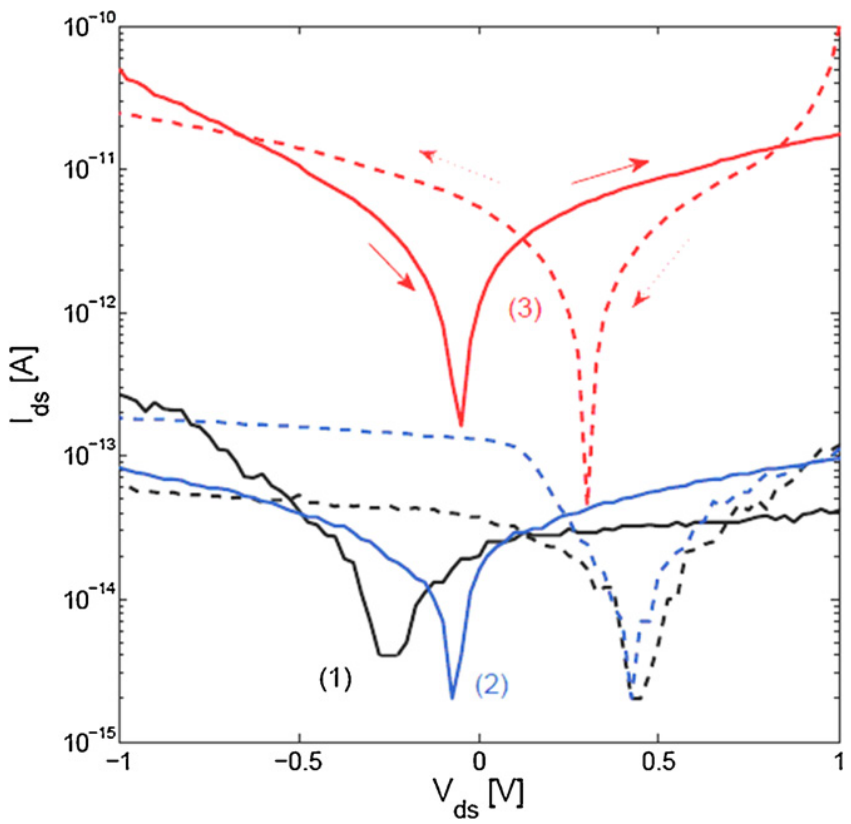

Fig. 10. Current minima gaps for $-1 \mathrm{~V} \leq V_{\mathrm{ds}} \leq+1 \mathrm{~V}$ clearly showing the trend of memristive-biosensors behavior with increasing antigen concentration: (1) $0 \mathrm{fM}$, (2) $5 \mathrm{fM}$, (3) $10 \mathrm{fM} \mathrm{AAB}$ solution. Arrows indicate sweep directions. with an increasing amount of antigen uptake has been verified with all the realized and tested memristive-biosensors. So, we can define the detection parameter:

$D=-\Delta V_{\mathrm{ds}}=-\left(\left.V_{\mathrm{ds}}\right|_{\text {backward-min }}-\left.V_{\mathrm{ds}}\right|_{\text {forward-min }}\right)$

Fig. 11 shows the calibration curve on this parameter. The collected data are positioned along a quite linearly relationship. We acquired similar and reproducible behaviors on many sensors. By following

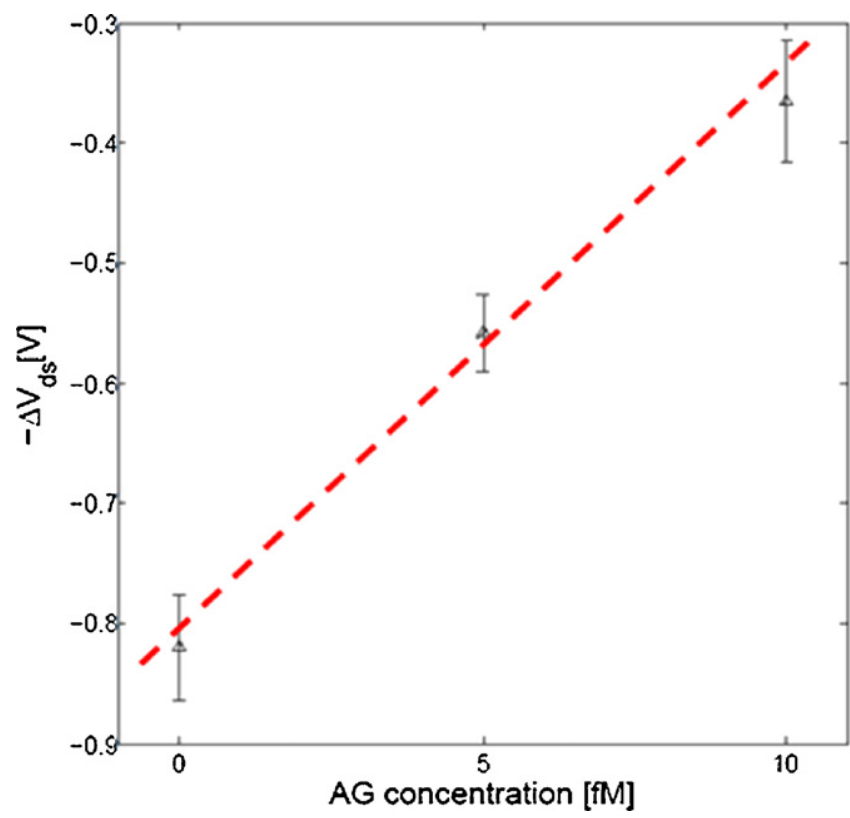

Fig. 11. Calibration curve obtained for three $A A B$ concentrations. The up-take with the $A B$ layer attached to the Si nanowire modifies the memristive behavior such that $-\Delta V_{\mathrm{ds}}$ increases after wetting with more concentrated $\mathrm{AAB}$ solutions. 


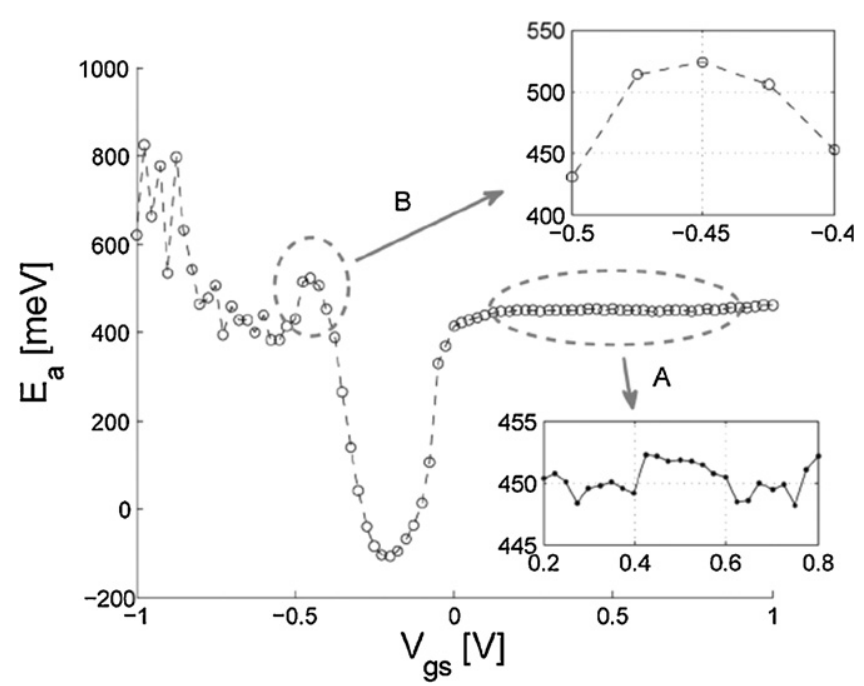

Fig. 12. Activation energy plot showing the extraction of Schottky barrier height on freestanding channels with all-around-gate demonstrating a Schottky barrier height of $525 \mathrm{meV}$.

the usually accepted IUPAC definitions for biosensors [28], we can write the sensitivity as:

$S=\frac{\left.D\right|_{C_{2}}-\left.D\right|_{C_{1}}}{C_{2}-C_{1}}$

where the $\left.D\right|_{C_{x}}$ is the detection parameter at the concentration $C_{x}$, and $C_{2}, C_{1}$ are two different antigens concentration. We can also define the limit of detection as:

$\mathrm{LOD}=\frac{k \Delta D}{S}$

where $\Delta D$ is the measure of blank, $S$ is the sensitivity, and $k$ returns the statistical significance ( $k=1,2$, or 3 means $68.2 \%, 95.4 \%$, or $99.6 \%$ of statistical confidence). By Eqs. (2) and (3), the performances of our memristive-biosensors are estimated in a sensitivity equal to $37 \pm 1 \mathrm{mV} / \mathrm{fM}$ and a limit of detection equal to $3.4 \pm 1.8 \mathrm{fM}$.

\subsection{The role of virtual gate and Schottky barrier}

The un-functionalized Si nanowire devices show a typical memristive $I_{\mathrm{ds}}-V_{\mathrm{ds}}$ behavior with zero-crossing property at $V_{\mathrm{ds}}=0 \mathrm{~V}$. Upon functionalization, the forward and reverse voltage sweeps split, originating a hysteresis where the zero-current condition occurs for different and non-zero $V_{\mathrm{ds}}$ values, as clearly demonstrated in Section 3.2. This effect is due to simultaneous occurrence of molecule gating effects and Schottky-barrier lowering at the silicide-to-Si junctions. Accordingly, $I_{\mathrm{ds}}-V_{\mathrm{ds}}$ curves show an increase of current conductance as well as an enlargement of the hysteresis window, which is due to the presence of charged molecules, e.g. the antibodies, around the freestanding channel. The temperature also induces a change of the current conductance in memristive devices [29]. By means of the activation energy, the relationship between current conductivity and temperature give us information about the Schottky barrier height of our memristivebiosensors. However, we cannot easily perform the measures of the activation energy on the freestanding structures with the biological functionalization. This is related to problems of different parasitic capacitances obtained on the same pad as due to sample removing form the probe station for steps of wetting, washing, and drying (see Section 3.2). Therefore, the current-temperature measurements are performed on fully passivated structures that have the silicon all-around gates (Fig. 9B). Fig. 12 shows the graph of

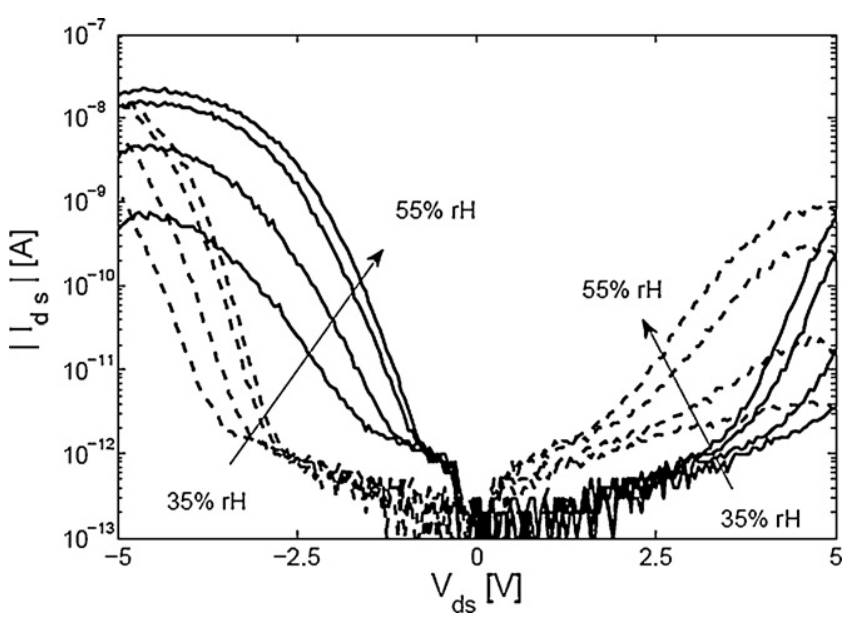

Fig. 13. Effect of increasing air humidity on the $I_{\mathrm{ds}}-V_{\mathrm{ds}}$ curves taken at $T=22^{\circ} \mathrm{C}$. The curves are taken within a $40 \mathrm{~min}$ time frame and follow an humidity gradient induced by inserting a water tank inside the measurement chamber. Continuous sweeps taken every $10 \mathrm{~min}$ confirm the increase of current upon increasing air humidity levels along with rH saturation at $55 \%$.

the acquired activation energy plotted versus the $V_{\text {gs }}$. The Schottky barrier may be obtained by using the equation [30]:

$I=A A^{*} T^{2} e^{-q \phi_{b} / k T}\left[e^{q(V-I R) / n T}-1\right]$

where $\phi_{b}$ is the Schottky barrier, $T$ is the temperature, $A^{*}$ is the Richardson constant, $A$ the area of the Schottky junction, $q$ the electron charge, and $n$ the ideality factor of the junction. If we only need to estimate the Schottky barrier, we can acquire the current at different temperatures and plot the $\log$ of resistivity versus $1 / T$ [31]. The fitting on this plot returns the activation energy. Fig. 12 maps this energy at different $V_{\mathrm{gs}}$ potentials. The estimation with this method gives us a junction with mid-gap Schottky barrier $\phi_{b} \approx 525 \mathrm{meV}$.

\subsection{The effect of the humidity}

Eq. (4) shows that the current is affected by temperature. The current properties of dried organic samples are affected by humidity [32], too. Water molecules adsorbed from humid environment into thin organic films have been measured with both hydrophilic [34] and hydrophobic [33] molecules. Therefore, all the data we reported in Sections 3.2 and 3.3 were acquired in highly controlled conditions dealing with temperature and humidity. After functionalization, the devices are rinsed for $5 \mathrm{~min}$ in de-ionized water. Then the devices are dried under nitrogen flow. Before measuring, the devices are left inside a sealed measurement chamber maintained at fixed temperature and humidity for about 20 min. This operation is performed to get all the devices into steady equilibrium condition with the chamber. To further investigate the effect of air relative humidity $(\mathrm{rH})$, we introduced a wet towel inside the chamber for measuring the $\mathrm{rH} \%$ together with $I_{\mathrm{ds}}-V_{\mathrm{ds}}$ curves. As shown in Fig. 13, rH\% increases from 31\% to 55\% within 40 min meanwhile the memristive conductivity does change. Fig. 13 shows that the change in relative humidity affects the current along the channel. Moreover, it hides the voltage gap, too. The detection parameter $\mathrm{D}$ is confused within a dynamic range of more than $1 \mathrm{~V}$ due to the changes in relative humidity. This proofs that the charges of water molecules acts on the virtual gate voltage as well as those of antibodies. The water affects the memristive behavior of our freestanding channels, too. This phenomenon of water charging effect is also observed in carbon nanotubes lying on $\mathrm{SiO}_{2}$ surfaces and working in ambient air. In fact, hysteresis on the channel current is registered in FET transistors made with carbon 
nanotubes as related to the humidity of the air [35]. In that case, the hysteresis on the FET electrical properties is due to trapping of water molecules around carbon nanotubes. The hysteresis is verified to persist even in dried air. This confirms that the water is enough strongly trapped onto our surface of silicon di-oxide as well as in that of carbon nanotubes. This is assured by electrostatic interactions between the oxygen and hydrogen atoms that are present in both water molecules and carbon or silicon surfaces (see Fig. 2 in Ref. [35]). On carbon nanotubes, a current variation up to $60 \mathrm{nA}$ is registered in air for an applied $V_{\mathrm{gs}}$ equal to $-5 \mathrm{~V}$ (Fig. 4(a) in Ref. [35]), while no variations are registered in vacuum (Fig. 3(b) in Ref. [35]). Even if the material of our freestanding channel is not the same of that reference (we are using silicon and silicon nitride instead of carbon nanotubes), the currents registered in Fig. 13 at $-5 \mathrm{~V}$ confirms the same range of variations (order of magnitudes in tens of $\mathrm{nA}$ ) ranging from $35 \%$ up to $55 \%$ in $\mathrm{rH}$. A further confirmation in the comparison comes by the voltage gap. The hysteresis on $V_{\mathrm{gd}}$ upon a rH variation from $35 \%$ up to $60 \%$ is in the range of $1 \mathrm{~V}$ in the case of FET based on carbon nanotubes (Fig. 5(d) of Ref. [35]). Similarly, the noise we registered in the voltage-gap in Fig. 13 is in the same voltage range for similar range of relative humidity.

Taking into account the device sensitivity to air $\mathrm{rH} \%$ (this section) and to temperature (Section 3.4), all the measurements reported in Sections 3.2 and 3.3 have been carried out at $22^{\circ} \mathrm{C}$ and $31 \% \mathrm{rH}$.

\section{Conclusions}

In this paper, we have seen that freestanding channels realized in silicon manifest memristive behavior useful to obtain memristive-biosensors.

The registered memristive behavior shows a clear voltage gap between the current minima reached in forward and backward branches of the $I_{\mathrm{ds}}-V_{\mathrm{ds}}$ curves. The memristive hysteresis is acquired on structures functionalized with antibodies under dried conditions in ambient air. This phenomenon is demonstrated being related to electrical charges of antibodies, which act as a virtual gate giving similar effects that those provided by all-around inorganic gates observed in fully passivated nano-structures. In particular, the phenomenon is related to the amount of injected charged Nickel atoms from the $\mathrm{Ni} / \mathrm{Li}$ metallic layer. The carriers' injection is modified by the electrical potential across the channel and affects the Schottky barrier at the interface. The Schottky barrier of the freestanding channels with all-around-gates is measured equal to $525 \mathrm{meV}$ by means of activation energy plots. The carriers' injection and, then, the Schottky barrier are further modified by the surface potential of antibodies.

The memristive behavior observed in the voltage gap is largely affected by antigen uptake. The calibration curves acquired for different amount of antigens concentration return an average sensitivity and limit of detection of $37 \pm 1 \mathrm{mV} / \mathrm{fM}$ and $3.4 \pm 1.8 \mathrm{fM}$, respectively.

The role of air humidity is also investigated. The humidity affects the conductivity in a manner that hide the voltage gap by increasing the noise in current minima. The variation in conductivity as due to the ambient humidity is compared with that on carbon nanotubes onto silicon di-oxide when used in ambient air. Indeed, a deeper understanding on how the humidity as affecting the voltage gap is still under discussion. More experiments and further work on the modeling are still required for a full understanding of all the registered properties.

In conclusion, we have in this paper the first demonstration of the possibility to exploit memristive behavior in freestanding nanowires for biosensing purposes on dried biological films. So, the paper reports the realization of a memristive biosensor.

\section{Acknowledgments}

The authors thank Pietro Delmastro for assistance in fabrication of the memristive devices. The authors also thank Fabienne Bobard for the acquisition of SEM images. Ioannis Xenarios and Akshat Dave are acknowledged for stimulating and very useful discussions. The work was supported by the multidisciplinary FNS grant (CR32I3_135073). This work has been also partially supported by the Swiss NSF grants no. 200021-122168, and no. 200021-132539, and by the Nano-Tera grant no. 20NA21-128841 and no. 20NA21128840 , evaluated by the Swiss NSF foundation.

\section{References}

[1] W.N. Frost, V.F. Castellucci, R.D. Hawkins, E.R. Kandel, Monosynaptic connections made by the sensory neurons of the gill- and siphon-withdrawal reflex in Aplysia participate in the storage of long-term memory for sensitization, Proceedings of the National Academy of Sciences of the United States of America 82 (23) (1985) 8266

[2] A. Lendlein, S. Kelch, Shape-memory polymers, Angewandte Chemie International Edition 41 (12) (2002) 2034.

[3] J.-M. Lehn, From supramolecular chemistry towards constitutional dynamic chemistry and adaptive chemistry, Chemical Society Reviews 36 (2) (2007) 151.

[4] L. Chua, Memristor - the missing circuit element, IEEE Transactions on Circuit Theory 18 (5) (1971) 507.

[5] D.B. Strukov, G.S. Snider, D.R. Stewart, R.S. Williams, The missing memristor found, Nature 453 (7191) (2008) 80.

[6] a.M.D.V. Yuriy, V. Pershin, Memory effects in complex materials and nanoscale systems, Advances in Physics 60 (2011) 145.

[7] M. Haykel Ben Jamaa, S. Carrara, J. Georgiou, N. Archontas, G. De Micheli, 9th IEEE Conference on Nanotechnology, 2009, IEEE-NANO 2009, 2009, p. 152.

[8] D. Sacchetto, M.H. Ben-Jamaa, S. Carrara, G. De Micheli, Y. Leblebici, Proceedings of 2010 IEEE International Symposium on Circuits and Systems (ISCAS), 2010 p. 9.

[9] D.B. Strukov, D.R. Stewart, J. Borghetti, X. Li, M. Pickett, G.M. Ribeiro, W. Robinett, G. Snider, J.P. Strachan, W. Wu, Q. Xia, J.J. Yang, R.S. Williams, Proceedings of 2010 IEEE International Symposium on Circuits and Systems (ISCAS), 2010, p. 1967.

[10] T. Berzina, A. Smerieri, M. Bernabo, A. Pucci, G. Ruggeri, V. Erokhin, M.P. Fontana, Optimization of an organic memristor as an adaptive memory element, Journal of Applied Physics 105 (12) (2009) 124515.

[11] T.W.K. Dong Ick Son, J.H. Shim, J.H. Jung, D.U. Lee, J.M. Lee, W.I. Park, W.K. Choi, Flexible organic bistable devices based on graphene embedded in an insulating poly(methyl methacrylate) polymer layer, Nano Letters 10 (7) (2010) 2441.

[12] M. Laiho, E. Lehtonen, 12th International Workshop on Cellular Nanoscale Networks and Their Applications (CNNA), 2010, p. 1

[13] J. Borghetti, Z. Li, J. Straznicky, X. Li, D.A.A. Ohlberg, W. Wu, D.R. Stewart R.S. Williams, A hybrid nanomemristor/transistor logic circuit capable of selfprogramming, Proceedings of the National Academy of Sciences of the United States of America 106 (6) (2009) 1699.

[14] T.C. Sung Hyun Jo, I. Ebong, B.B. Bhadviya, P. Mazumder, W. Lu, Nanoscale memristor device as synapse in neuromorphic systems, Nano Letters 10 (2010) 1297.

[15] F. Alibart, S. Pleutin, D. Guérin, C. Novembre, S. Lenfant, K. Lmimouni, C. Gamrat, D. Vuillaume, An organic nanoparticle transistor behaving as a biological spiking synapse, Advanced Functional Materials 20 (2010) 330-334.

[16] V. Erokhin, T. Berzina, P. Camorani, A. Smerieri, D. Vavoulis, J. Feng, M.P. Fontana, Material memristive device circuits with synaptic plasticity: learning and memory, BioNanoScience 1 (2011) 24-30.

[17] B. Wang, L.F. Zhu, Y.H. Yang, N.S. Xu, G.W. Yang, Fabrication of a $\mathrm{SnO}_{2}$ nanowire gas sensor and sensor performance for hydrogen, The Journal of Physical Chemistry C 112 (17) (2008) 6643.

[18] G. Zheng, F. Patolsky, Y. Cui, W.U. Wang, C.M. Lieber, Multiplexed electrical detection of cancer markers with nanowire sensor arrays, Nature Biotechnology 23 (10) (2005) 1294

[19] J.-i. Hahm, C.M. Lieber, Direct ultrasensitive electrical detection of DNA and DNA sequence variations using nanowire nanosensors, Nano Letters 4 (1) (2004) 51.

[20] V. Bhalla, S. Carrara, C. Stagni, B. Samorì, Chip cleaning and regeneration for electrochemical sensor arrays, Thin Solid Films 518 (12) (2010) 3360.

[21] Y. Yao, Y.-Z. Ma, M. Qin, X.-J. Ma, C. Wang, X.-Z. Feng, NHS-ester functionalized poly(PEGMA) brushes on silicon surface for covalent protein immobilization, Colloids and Surfaces B: Biointerfaces 66 (2) (2008) 233.

[22] A.J.W. Kusnezow, A. Walijew, F. Diehl, J. Hoheisel, Antibody microarrays: an evaluation of production parameters, Proteomics 3 (3) (2003) 254. 
[23] H.G.L.D. Kim, H. Jung, S.H. Kang, Single-protein molecular interactions on polymer-modified glass substrates for nanoarray chip application using dualcolor TIRFM, Bulletin of The Korean Chemical Society 28 (5) (2007) 783.

[24] E.O. Saphire, P.W.H.I. Parren, R. Pantophlet, M.B. Zwick, G.M. Morris, P.M. Rudd, R.A. Dwek, R.L. Stanfield, D.R. Burton, I.A. Wilson, Crystal structure of a neutralizing human IgG against HIV-1: a template for vaccine design, Science 293 (5532) (2001) 1155

[25] S. Carrara, V. Bhalla, C. Stagni, L. Benini, A. Ferretti, F. Valle, A. Gallotta, B. Riccò, B. Samorì, Label-free cancer markers detection by capacitance biochip, Sensors and Actuators B: Chemical 136 (2009) 163-172.

[26] L.O. Chua, S.M. Kang, Memristive devices and systems, Proceedings of the IEEE 64 (2) (1976) 209-223.

[27] D. Sacchetto, M.H. Ben-Jamaa, G. De Micheli, Y. Leblebici, Proceedings of the European Solid State Device Research Conference, 2009, ESSDERC'09, 2009, p. 245.

[28] A.D. Mc Naught, A. Wilkinson (Eds.), IUPAC. Compendium of Chemical Terminology, Blackwell Scientific Publications, Oxford, 1997.

[29] V. Allodi, V. Erokhin, M.P. Fontana, Effect of temperature on the electrical properties of an organic memristive device, Journal of Applied Physics 108 (2010), 074510-1, 7.

30] D. Sacchetto, G. De Micheli, Y. Leblebici, Ambipolar Si nanowire field effect transistors for low current and temperature sensing, in: Transducers 2011, Beijing, China, 2011.

[31] A.T.P.A.C. Schmitz, M. Asif Khan, Q. Chen, J.W. Yang, I. Adesida, Schottky barrier properties of various metals on n-type GaN, Semiconductor Science and Technology 11 (1996) 1464

[32] A.V.D. Abhishek Motayed, S.N. Mohammad, J. Melngailis, Experimental investigation of electron transport properties of gallium nitride nanowires, Journal of Applied Physics 104 (2008) 024302.

[33] S. Carrara, V. Erokhin, C. Nicolini, STM image formation of organic thin films: the role of water shell, Langmuir 16 (16) (2000) 6577.

[34] S. Carrara, L. Benini, V. Bhalla, C. Stagni, A. Ferretti, A. Cavallini, B. Riccò, B. Samorì, New insights for using self-assembly materials to improve the detection stability in label-free DNA-chip and immuno-sensors, Biosensors and Bioelectronics 24 (12) (2009) 3425.

[35] W. Kim, A. Javey, O. Vermesh, Q. Wang, Y. Li, H. Dai, Hysteresis caused by water molecules in carbon nanotube field-effect transistors, Nano Letters 3 (2) (2003) 193.

\section{Biographies}

Sandro Carrara is a lecturer at the EPFL in Lausanne and professor of optical and electrical biosensors at the Department of Electrical Engineering and Biophysics (DIBE) of the University of Genoa. His scientific interests are on electrical phenomena of nano-bio-structured films, with special focus on protein and DNA biochips. He has more then 100 scientific publications and 10 patents. His work has been awarded several times: at the Nanotera workshop in 2011 (Bern), at the NanoEurope Symposium in 2009 (Rapperswil), at the IEEE International Conference PRIME with the bronze leaf prize in 2010 (Berlin), and with the golden leaf prize in 2009 (Cork) In 2006, he received the best referees' award from Biosensor and Bioelectronics journal. He also won a NATO Advanced Research prize in 1996 for his original contribution to the physics of single-electron conductivity in nano-particles. He is Founde and Editor-in-Chief of the journal BioNanoScience by Springer, and Associate Editor of two IEEE Transactions. He is in several Boards of International Conferences on Circuits and Systems for Biomedical Applications.
Davide Sacchetto received the B.S. degree in physics engineering from Politecnico di Torino (Italy) in 2007. In 2008 he received the jointed M.S. degree in micro and nano technologies for integrated systems from École Polytechnique Fédérale de Lausanne, the Institut National Polytechnique de Grenoble and the Politecnico di Torino. Since November 2008 he is working as a Ph.D. student at the Microelectronic System Laboratory (LSM) and the Integrated System Laboratory (LSI), EPFL. His research interests focus on novel devices, investigating issues ranging from solid-state microfabrication to circuit implementation.

Marie-Agnès Doucey has a Ph.D. in biochemistry received from the FriedrichMiescher Institute in Novartis, Basel (Switzerland) passed with distinction (mention très honorable). She also holds a degree in Engineer in Biotechnology received at the "Ecole Supérieure de Biotechnologies de Strasbourg" (E.S.B.S), France. She is now a project leader at the DEO (Division of Experimental Oncology) in the University of Lausanne/CHUV, Lausanne. She was a project leader at the DEO (Division of Experimental Oncology) of the University of Lausanne/CHUV/CePO, and "Maitreassistante" in the Institute of Biochemistry, in Epalinges. She has more than twenty publications in peer-reviewed international journals.

Camilla Baj-Rossi received a bachelor degree in 2008 and a master degree in 2010 in biomedical engineering at Politecnico di Torino (Italy). She carried out her master project at EPFL in the Integrated System Laboratory (LSI), working on the design of a multi-panel biochip based on cytochromes and carbon nanotubes for drugs monitoring in personalized therapy. Currently she is a Ph.D. student in microsystems and microelectronics in the Integrated System Laboratory (LSI) under the direction of Prof. Giovanni De Micheli and Sandro Carrara. The aim of her Ph.D. thesis is the design of a multi-panel biochip based on nanostructures for the electrochemical detection of multiple drug compounds in treatments of several diseases.

Giovanni De Micheli is a professor and director of the Institute of Electrical Engineering and of the Integrated Systems Centre at EPFL, Switzerland. He is the program leader of the Nano-Tera.ch program. He is a fellow of ACM and IEEE and a member of the Academia Europaea. His research interests include several aspects of design technologies for integrated circuits and systems, such as synthesis for emerging technologies, networks on chips and 3D integration. He is also interested in heterogeneous platform design including electrical components and biosensors, as wel as in data processing of biomedical information. He is the recipient of the 2003 IEEE Emanuel Piore Award for contributions to computer-aided synthesis of digital systems. He received the Golden Jubilee Medal for outstanding contributions to the IEEE CAS Society in 2000 . He received the 1987 D. Pederson Award for the best paper on the IEEE Transactions on CAD/ICAS, two Best Paper Awards at the Design Automation Conference, in 1983 and in 1993, and a Best Paper Award at the DATE Conference in 2005

Dr. Yusuf Leblebici is a chair professor at the Swiss Federal Institute of Technology in Lausanne (EPFL), and director of Microelectronic Systems Laboratory. His research interests include design of high-speed CMOS digital and mixed-signal integrated circuits, computer-aided design of VLSI systems, intelligent sensor interfaces, modeling and simulation of semiconductor devices, and VLSI reliability analysis. $\mathrm{He}$ is the coauthor of 4 textbooks, namely, Hot-Carrier Reliability of MOS VLSI Circuits (Kluwer Academic Publishers, 1993), CMOS Digital Integrated Circuits: Analysis and Design (McGraw Hill, 1st Edition 1996, 2nd Edition 1998, 3rd Edition 2002), CMOS Multichannel Single-Chip Receivers for Multi-Gigabit Optical Data Communication (Springer, 2007) and Fundamentals of High Frequency CMOS Analog Integrated Circuits (Cambridge University Press, 2009), as well as more than 200 articles published in various journals and conferences. He has served as an associate editor of IEEE Transactions on Circuits and Systems (II), and IEEE Transactions on Very Large Scale Integrated (VLSI) Systems 